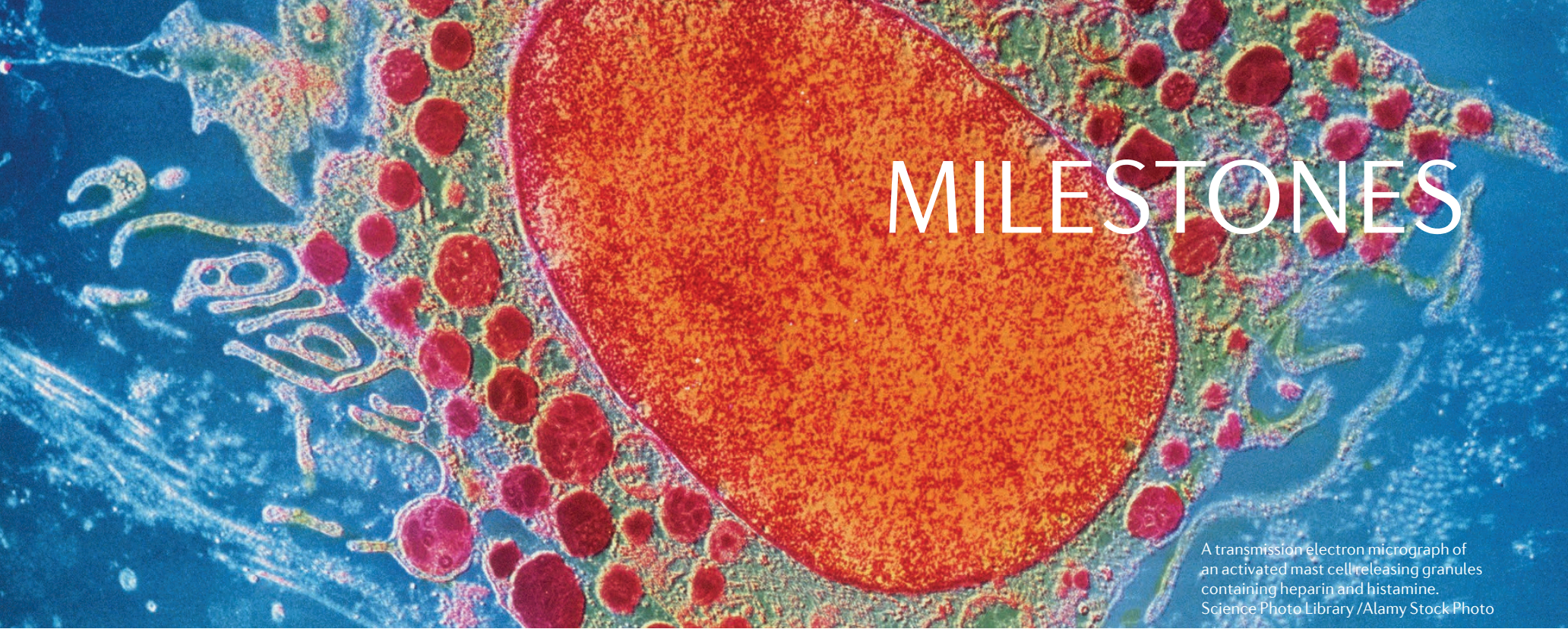

$\Rightarrow$ MILESTONE 1

\title{
Discovery and purification of heparin
}

Heparin was the first anticoagulant agent to be discovered and isolated for medical use, and is one of the oldest drugs still to be in widespread clinical use. Indeed, heparin remains on the WHO Model List of Essential Medicines - the safest and most effective medicines needed in a health-care system.

Heparin is a naturally occurring glycosaminoglycan produced in the body by basophils and mast cells (image). The substance was identified a centenary ago, although who should be credited with the discovery remains controversial.

In 1916, Jay McLean was a second-year medical student working with the physiologist William Henry Howell at Johns Hopkins Medical School in Baltimore, Maryland, USA The pair were initially working on cephalin, thought to be a procoagulant substance that neutralized antithrombin and thereby allowed the activation of prothrombin, leading to clotting.

After this work, McLean extracted fatsoluble compounds called phosphatides from dog liver that seemed to have anticoagulant properties in vitro, and which produced excessive bleeding when given to experimental animals. McLean then moved to the University of Pennsylvania and continued his research into cephalins.

Nevertheless, work on anticoagulants continued in the Howell laboratories. In 1918, together with medical student L. Emmett Holt Jr, Howell isolated another fat-soluble anticoagulant, distinct from the one previously isolated by McLean. Howell coined the name 'heparin' for this type of substance (derived from the Greek for 'liver', from which it was first isolated).
In 1922, Howell described an aqueous extraction protocol and, in 1926, refined this protocol and identified a water-soluble polysaccharide anticoagulant, which he also termed 'heparin' (despite being different from the compounds previously isolated in 1916 and 1918).

\section{4 heparin infused into the brachial artery resulted in a significantly increased clotting time}

This water-soluble heparin was commercially produced, but contained impurities that caused adverse effects such as headaches, fevers, and nausea, which limited its medicinal use. Howell retired in 1931, and died in 1945.

In 1929, Charles Best (famous for being a co-discover of insulin with fellow Canadian Sir Frederick Banting) working with graduate student Arthur Charles decided to try to purify heparin further to reduce or eliminate the adverse effects, and to demonstrate its utility in the prevention of thrombus formation. In 1933, Arthur Charles and senior colleague David Scott published a series of three papers outlining a protocol for isolating a crude preparation of heparin from bovine liver, an analysis of extrahepatic tissues in which heparin could be identified, and a protocol for purifying heparin.

In 1937, Best and colleagues published their observations that heparin prevented thrombus formation in dogs whose veins had undergone mechanical or chemical trauma. On 16 April 1937, the purified form of heparin was used in a human for the first time: a saline solution of heparin infused into the brachial artery resulted in a significantly increased clotting time, with no toxic adverse effects.

A Swedish physiologist Erik Jorpes had visited Best in Canada in 1929 and then returned to the Karolinska Institute in Stockholm. In 1935, Jorpes published his research into the structure of heparin, which allowed a Swedish company to begin commercial production of heparin for intravenous use. By 1949, Peter Moloney and Edith Taylor had patented a method to produce heparin with a high yield and at a low cost, which established the widespread availability and use of the drug.

Before the 1940s, Howell was widely credited with the discovery of heparin, although Best and many others contributed to its development into a clinically usable product. In 1963, a plaque was unveiled at Johns Hopkins University to commemorate Jay McLean MD (1890-1957), "in recognition of his major contribution to the discovery of heparin in 1916 as a second-year medical student in collaboration with Professor William H. Howell”.

Gregory B. Lim, Chief Editor, Nature Reviews Cardiology

\footnotetext{
ORIGINAL ARTICLES Howell, W. H. \& Holt, E. Two new factors in blood coagulation - heparin and pro-antithrombin. Am.J. Physiol. 47, 328-341 (1918) |Charles, A. F. \& Scott, D. A. Studies on heparin: I. The preparation of heparin.J. Biol. Chem. 102, 425-429 (1933) |Charles, A. F. \& Scott, D. A. Studies on heparin: II. Heparin in various tissues. J. Biol. Chem. 102, 431-435 (1933) Charles, A. F. \& Scott, D. A. Studies on heparin. III. The purification of heparin. J. Biol. Chem. 102,437-448 (1933)| Murray, D. W. G. et al. Heparin and the thrombosis of veins following injury. Surgery 2, 163-187 (1937) | Jorpes, E. The chemistry of heparin. Biochem.J. 29, 1817-1830 (1935). FURTHER READING Wardrop, D. \& Keeling, D. The story of the discovery of heparin and warfarin. Br.J. Haematol. 141, 757-763 (2008)
} 